\title{
Short-term, Low-Dose Etoposide in Refractory Adult- Onset Still's Disease-Associated Macrophage Activation Syndrome, a Case Series Study
}

\author{
Ran Wang \\ Renji Hospital
}

Ting Li

Renji Hospital

Shuang Ye

Renji Hospital

Liangjin Lv

Renji Hospital

Sheng Chen

Renji Hospital

Xiaodong Wang

Renji Hospital

Chun-de Bao

Renji Hospital

Qiong Fu ( $\nabla$ fuqiong@renji.com )

Renji Hospital

\section{Research Article}

Keywords: adult-onset Still's disease, macrophage activation syndrome, etoposide

Posted Date: December 10th, 2021

DOI: https://doi.org/10.21203/rs.3.rs-1129785/v1

License: (1) (1) This work is licensed under a Creative Commons Attribution 4.0 International License. Read Full License 


\section{Abstract}

\section{Objectives:}

In this study, we modified the classical regimen of the hemophagocytic lymphohistiocytosis-04 protocol and evaluated the efficacy and safety of short-term, low-dose etoposide in patients with refractory macrophage activation syndrome (MAS) associated with adult-onset Still's disease (AOSD).

\section{Methods:}

A total of 17 patients with refractory AOSD-associated MAS were enrolled and received short-term, lowdose etoposide (100 mg twice a week for four times). Another 11 patients, who were not treated with etoposide, were included as historical controls. Patient information, such as clinical manifestations, laboratory results, treatments, and short-term prognosis, were recorded and analyzed.

\section{Results:}

In this case series, $88.24 \%$ of the patients with MAS who were treated with short-term, low-dose etoposide had a favorable response in 3 weeks, which was significantly higher $(p=0.017)$ than that in the patients with MAS who were treated without etoposide (45.45\%). The 90-day survival rate after the onset of MAS was significantly higher $(p=0.0029)$ among the patients in the short-term etoposide group $(16 / 17$, $94.12 \%)$ than in the control group (5/11, $45.45 \%)$.

\section{Conclusion:}

The regimen of short-term (2-week), low-dose etoposide was highly effective in the treatment for patients with refractory AOSD associated MAS with an acceptable safety profile.

\section{Introduction}

Adult-onset Still's disease (AOSD) is a rare autoinflammatory disease with heterogeneous clinical presentation and nonspecific features (spiking fever, pharyngitis, arthritis, and skin rash with elevated acute-phase reactants) ${ }^{[1]}$. Macrophage activation syndrome (MAS) is a life-threatening complication that occurs in $10-19 \%$ of patients with AOSD (hereafter referred to as AOSD-MAS), with a short-term mortality rate as high as $20 \%{ }^{[2]}$.

MAS falls under the umbrella term "cytokine storm syndrome". It is also known as secondary hemophagocytic lymphohistiocytosis $(\mathrm{HLH})^{[3]}$. Connetive tissue disease related MAS most frequently occurs in patients with AOSD, systemic juvenile idiopathic arthritis (sJIA), and systemic lupus erythematosus $^{[4]}$.

Currently, there is no high level evidence to guide the management of AOSD-MAS, probably because of its rarity. Recent years have seen several case series and clinical trials supporting the use of the IL-1 
antagonist anakinra in the treatment of MAS complicated with SJIA or AOSD ${ }^{[5]}$. IL-18 antagonists, IFN- $\gamma$ inhibition, and the JAK inhibitor ruxolitinib are also promising treatment options ${ }^{[6]}$; Anti-IL-6 treatment of MAS is controversial ${ }^{[7]}$. While these novel biologics might be effective against MAS, many of them have not been approved ${ }^{[8]}$. Accessibility and affordability are the main concerns that prevent the widespread implementation of biologics in the treatment of adult MAS in current clinical practice.

The HLH-2004 protocol should be considered for patients with AOSD-MAS who inadequately respond to high doses of steroids ${ }^{[9]}$. The HLH-04 protocol, which combines etoposide, dexamethasone, and cyclosporine, was initially developed for pediatric patients with primary $\mathrm{HLH}^{[10]}$. Although the $\mathrm{HLH}-04$ protocol is highly effective, the original pediatric protocol would result in overtreatment and increased toxicity in adults, limiting its application in patients with AOSD-MAS ${ }^{[11]}$. Although dose reductions and individualized tailoring of treatment are recommended for adult patients with $\mathrm{MAS}^{[11-13]}$, there is still a lack of reports to guide the application of the HLH-04 protocol in these patients.

In the current study, we sought to explore and evaluate a treatment strategy of revised HLH-04 regimen for patients with AOSD-MAS who failed to respond to an initial high-dose steroid treatment.

\section{Methods}

\section{Patients}

Patients were included in this study if they were older than 18 years of age; met the Yamagishi criteria for the diagnosis of AOSD and the HLH-2004 criteria for MAS; and were unresponsive to or dependent on a steroid dosage equivalent to methylprednisolone $2 \mathrm{mg} / \mathrm{kg} /$ day for 3 days. Patients were excluded if they had other connective tissue diseases, underlying cancer, or a concomitant infection. From January 2015 to June 2020, a total of 17 patients were enrolled at our center and received short-term, low-dose etoposide (100 mg biw four times) combined with dexamethasone (equivalent to prednisolone 1$2 \mathrm{mg} / \mathrm{kg} /$ day), followed by oral calcineurin inhibitor and glucocorticoid tapering. From January 2012 to December 2014, another 11 patients, who were treated without etoposide, were included as historical controls.

This study was approved by the Shanghai Renji Hospital Ethics Committee (No. 259 2013-126). The study was conducted in accordance with the principles of the Declaration of Helsinki. All patients signed written consent forms.

\section{Data collection}

Medical charts were reviewed by three rheumatologists. The following clinical features were also recorded at admission: fever, rash, joint pain, pharyngitis, myalgia, and abdominal pain. Laboratory test results at the time of MAS diagnosis were collected, including routine blood tests, liver function tests, renal function tests, and coagulation tests, as well as tests for fibrinogen, C-reactive protein, the erythrocyte sedimentation rate, ferritin, and triglycerides. We also recorded the clinical manifestations on 
day 7 and 21 after treatment. Other examination results included a bone marrow smear and bone marrow biopsy, ultrasound, computed tomography, and positron emission computed tomography; other related examination items were also collected when available. The use of medications, including glucocorticoids, immunosuppressive agents, or intravenous immunoglobulin, was also recorded. The 90-day survival rate after the onset of MAS was collected.

\section{Response}

The overall response included the percentage of patients with a complete response (CR), partial response (PR), or improvement in measures of HLH or MAS. A CR was defined as no fever, a normal spleen size, no cytopenia, no hyperferritinemia (defined as a ferritin level of $>2,000 \mathrm{ng} / \mathrm{mL}$ ), no evidence of coagulopathy, no neurologic or cerebrospinal fluid abnormalities attributable to $\mathrm{HLH}$, and no sustained increase in the level of soluble CD25. A PR was defined as three or more HLH-associated clinical and laboratory abnormalities that met the criteria for a CR. Improvement in measures of HLH was defined as a change of $>50 \%$ from baseline in at least three $\mathrm{HLH}$-associated clinical and laboratory abnormalities that were not normalized $^{[14]}$.

\section{Statistical analysis}

Clinical data were analyzed using SPSS 21 and Prism 7.0.1. Clinical features before and after treatment were analyzed. Paired $t$-test was used for data conforming to normal distribution, and paired nonparametric test was used for data not conforming to normal distribution. Quantitative data are presented as medians with interquartile ranges, and categorical data are presented as absolute numbers with percentages and $p$-values. A two-sided test $p$ value of $<0.05$ was considered statistically significant.

\section{Results}

\section{Demographic information and clinical characteristics}

All of the patients included in this study had uncontrollable MAS (either unresponsive or dependent) on a steroid dosage equivalent to methylprednisolone $2 \mathrm{mg} / \mathrm{kg} /$ day for at least 3 days.

For the 17 patients, short-term (2-week), low-dose etoposide was added to the therapy. In the 11 historical controls, the treatment included a large dose of steroids, calcineurin inhibitors, and/or tocilizumab.

The demographic information and baseline characteristics of the patients are shown in Table 1. There were no significant differences in the demographic information and clinical characteristics between the two groups.

\section{Responses to the short-term, low-dose etoposide regimen}

The use of etoposide remarkably and quickly improved the clinical manifestations and laboratory results. In particular, the platelet counts and the levels of alanine aminotransferase (ALT), aspartate 
aminotransferase (AST), and ferritin considerably and quickly improved over time in the etoposide group (Figure 1).

At 21 days, a total of $88.24 \%$ (15/17) of the patients with AOSD-MAS who were treated with etoposide showed a response; among these patients, $11.76 \%$ (2/17) achieved improvement, $23.53 \%(4 / 17)$ achieved a PR, and 52.95\% (9/17) achieved a CR. Among the patients with AOSD-MAS who were treated without etoposide, $45.45 \%$ (5/11) had a response, of which $9.09 \%$ (1/11) achieved improvement, $18.18 \%$ $(2 / 11)$ achieved a PR, and $18.18 \%(2 / 11)$ achieved a CR.

Meanwhile, patients in etoposide group received significantly lower cumulative dosage as well as daily mean dosage of steroid compared to those without etoposide group (daily mean dose of steroid equivalent to methylprednisolone, $140 \mathrm{mg}$ vs. $200 \mathrm{mg}, p=0.002$ respectively). More details on the treatments in both groups were shown in supplementary materials.

\section{Survival analysis}

The 90-day survival rate after the onset of MAS was significantly higher $(p=0.0029)$ among the patients in the etoposide group $(16 / 17,94.12 \%)$ than among those in the control group $(5 / 11,45.45 \%)$. (Figure 1 )

\section{Side effects and complications of short-term, low-dose etoposide in the treatment of AOSD-MAS}

In the etoposide group, the disease remained uncontrolled in one patient (5.88\%), the pancytopenia rapidly progressed, leading to death on the 7th day after the diagnosis of AOSD-MAS. Two patients (15.38\%) experienced nosocomial infections after etoposide therapy and quickly improved after antibiotic treatment. In the control group, 5 of the 11 patients died because of uncontrolled MAS.

\section{Discussion}

MAS is a potentially life-threatening complication of AOSD that requires early recognition and urgent intervention. Currently, the diagnosis and treatment of AOSD-MAS are based on the studies and clinical practice in SJIA associated MAS ${ }^{[15]}$. AOSD resembles SJIA clinically and these two diseases are considered to represent the same disease continuum with different ages of onset. However, the triggers for MAS, organ reserve, fitness, and clinical presentation differ between the pediatric and adult age groups $^{[16]}$. Transferring the pediatric precepts such as PRINTO/EULAR/ACR SJIA-MAS classification criteria and treatment regimens to adult patients may not always be appropriate. In fact, the PRINTO sJIA associated MAS criteria showed a very low specificity in diagnosing MAS in AOSD patients ${ }^{[17]}$. In the current study, we used the classic HLH-04 criteria to diagnose MAS in AOSD patients. Bone marrow study was done in each patient and around $75 \%$ of them had evidence of hemophagocytic phenomenon. Therefore, the patients in the study were a group of severe MAS patients with a prominent hyperinflammation as well as remarkable immune cell dysfunction. 
Guideline for adult HLH recommended that large dose of glucocorticoids should be the first line treatment for MAS in adults. ${ }^{[11]}$ In daily clinical practice, most AOSD patients respond well to a dosage of prednisone $2-3 \mathrm{mg} / \mathrm{kg} / \mathrm{d}$ or less ${ }^{[18]}$. When AOSD patients enter into the stage of MAS, and have a lack of or inadequate response to the initial glucocorticoid treatment, should we increase the dosage of steroid to high-dose pulse methylprednisolone (for example: $500 \mathrm{mg} / \mathrm{d}-1 \mathrm{~g} / \mathrm{d}$ for 3-5 consecutive days), or should we start $\mathrm{HLH}-04$ protocol since all these patients met the criteria of $\mathrm{HLH}$ ? $^{[6,11,19]}$ In the current study, we found that adopting a revised $\mathrm{HLH}-04$ protocol in these AOSD associated MAS patients was very effective and safe. Compared to historic controls, patients in short-term (2 weeks), low dose etoposide group had significantly higher survival rate and quicker improvement in terms of clinical manifestations and laboratory results, as well as significantly less exposure to glucocorticoid. The safety profile was also acceptable in etoposide group, with 2 cases of infections that responded well to antibiotics. There was no case of long-term bone marrow suppression, liver or renal dysfunction. These results provided compelling evidence supporting that low dose etoposide should be considered in AOSD-MAS patients who failed an initial $2-3 \mathrm{mg} / \mathrm{kg} / \mathrm{d}$ dose of glucocorticoid.

Compared to the novel cytokine targeted treatments, such as IL-1\IL-6 inhibitors, IL-18 antagonists, IFN- $\gamma$ inhibition and JAK inhibitors, etoposide has much broader mechanisms of action and is irreplaceable in the treatment of HLH. Etoposide could inhibit T-cell proliferation with high specificity and reduce secretion of proinflammatory cytokines in mice ${ }^{[20]}$. Incorporation of etoposide in $\mathrm{HLH}-94$ or $\mathrm{HLH}-04$ regimen has significantly increased the disease response rate from less than $10 \%$ to approximately $70 \%{ }^{[11]}$. However, $\mathrm{HLH}$ regimens are rarely reported in the treatment of patients with AOSD-MAS. This is probably because the etiology and pathogenesis of AOSD associated MAS are quite complex, many of AOSD-MAS patients have a favorable response to steroid alone or anti-cytokine treatment, therefore, do not require an aggressive treatment of etoposide. Rheumatologists usually have little experience in using etoposide, physician's preference is also a critical reason for drug selection. In addition, most adult patients could not tolerate the high dosage of etoposide recommended in $\mathrm{HLH}-94$ or $\mathrm{HLH}-04$ regimens. Many adult patients may have chronic comorbidities that make them more vulnerable to end-organ damage caused by cytokine storm as well as to side effects by chemotherapy drugs.

In line with this, we revised the original $\mathrm{HLH}-04$ regimen, reduce the dosage of etoposide to $100 \mathrm{mg}$ twice a week for 2 weeks (comparable with the recommended dosage of $50-100 \mathrm{mg} / \mathrm{m}^{2} / \mathrm{w}$ in 2019 guideline for adult $\mathrm{HLH})^{[11]}$. The revised regimen preserved the efficacy of etoposide, and at the same time, notably decreased the side effects of etoposide. In addition, adding etoposide in the treatment could remarkably reduce the dosage of glucocorticoids, providing further benefits for AOSD associated MAS patients.

This study is a single-center case-series study. Prospective studies with larger sample sizes are needed to further evaluate the effectiveness of this low-dose etoposide regimen in patients with AOSD associated MAS. Second, we only analyzed a short-term outcome in the AOSD- MAS patients. A long-term follow-up is required in the future studies. 


\section{Conclusion}

The current study is, to our knowledge, the first report of applying short-term, low dose etoposide in the treatment of AOSD associated MAS. We found that short-term (2-week), low-dose etoposide was highly effective in patients with AOSD associated MAS, especially for those with severe and life-threatening disease, with an acceptable safety profile. The study provides evidence for an inexpensive, easily accessible, highly effective and standard regimen for managing AOSD associated MAS. Further studies are needed to determine whether this modified regimen would be effective and appropriate for the treatment of MAS complicated with lupus and other autoimmune and auto-inflammatory diseases.

\section{Abbreviations}

ALT: alanine aminotransferase; AOSD: adult-onset Still's disease; AST: aspartate aminotransferase; CR: complete response; HLH: hemophagocytic lymphohistiocytosis; MAS: macrophage activation syndrome; PR: partial response; sJIA: systemic juvenile idiopathic arthritis.

\section{Declarations}

\section{Ethical Approval and Consent to participate}

This study was approved by Shanghai Renji Hospital Ethics Committee (No.259 2013-126). The study was conducted in accordance with the principles expressed in the Declaration of Helsinki.

\section{Consent for publication}

Not applicable.

\section{Availability of supporting data}

All relevant data are contained within the paper.

\section{Competing interests}

The authors declare that they have no competing interests

\section{Funding}

This work is supported by Shanghai Municipal Science and Technology Fund 21ZR1438800, Shanghai Talents Development fund 2019092 and Shanghai Pujiang Young Rheumatologists Training Program fund SPROG201907.

\section{Authors' contributions}


Ran Wang, Ting Li, Chun-de Bao and Qiong Fu wrote the main manuscript text.Ran Wang,Ting Li, Shuang Ye, Liangjin Lv, Sheng Chen, and Xiaodong Wang provided patient information and complete the data analysis. All authors reviewed the manuscript.

\section{Acknowledgements}

Not applicable.

\section{References}

[1.]Balderas F, Casillas R, Saavedra E, et al. Adult-onset Still's disease. Best Practice \& Research Clinical Rheumatology 2014;13(5-6):708-722.

[2.]Guerrieri A, Angeletti G, Mazzolini M, et al. Pulmonary involvement in adult Still's disease: Case report and brief review of literature. Respiratory medicine case reports 2017;22:91-94. doi:

10.1016/j.rmcr.2017.07.001

[3.]Bracaglia C, Prencipe G, De Benedetti F. Macrophage Activation Syndrome: different mechanisms leading to a one clinical syndrome. Pediatric rheumatology online journal 2017;15(1):5. doi: 10.1186/s12969-016-0130-4

[4.]Kumakura S, Murakawa Y. Clinical characteristics and treatment outcomes of autoimmune-associated hemophagocytic syndrome in adults. Arthritis \& rheumatology 2014;66(8):2297-2307. doi:

10.1002/art.38672

[5.]Schulert GS, Grom AA. Pathogenesis of macrophage activation syndrome and potential for cytokinedirected therapies. Annu Rev Med 2015;66:145-159. doi: 10.1146/annurev-med-061813-012806

[6.]Yildiz H, Bailly S, Van Den Neste E, et al. Clinical Management of Relapsed/Refractory Hemophagocytic Lymphohistiocytosis in Adult Patients: A Review of Current Strategies and Emerging Therapies. Therapeutics and clinical risk management 2021;17:293-304. doi: 10.2147/TCRM.S195538 [published Online First: 2021/04/24]

[7.]Kaneko $\mathrm{Y}, \mathrm{Kameda} \mathrm{H}$, Ikeda $\mathrm{K}$, et al. Tocilizumab in patients with adult-onset still's disease refractory to glucocorticoid treatment: a randomised, double-blind, placebo-controlled phase III trial. Annals of the rheumatic diseases 2018;77(12):1720-1729. doi: 10.1136/annrheumdis-2018-213920 [published Online First: 2018/10/04]

[8.]Ahmed A, Merrill SA, Alsawah F, et al. Ruxolitinib in adult patients with secondary haemophagocytic lymphohistiocytosis: an open-label, single-centre, pilot trial. Lancet Haemato/ 2019 doi: 10.1016/S23523026(19)30156-5 [published Online First: 2019/09/21]

[9.]EhI S, Astigarraga I, von Bahr Greenwood T, et al. Recommendations for the Use of Etoposide-Based Therapy and Bone Marrow Transplantation for the Treatment of HLH: Consensus Statements by the HLH 
Steering Committee of the Histiocyte Society. J Allergy Clin Immunol Pract 2018;6(5):1508-1517. doi: 10.1016/j.jaip.2018.05.031 [published Online First: 2018/09/12]

[10.]Henter JI, Horne A, Arico M, et al. HLH-2004: Diagnostic and therapeutic guidelines for hemophagocytic lymphohistiocytosis. Pediatr Blood Cancer 2007;48(2):124-131. doi: 10.1002/pbc.21039

[11.]La Rosee P, Horne A, Hines M, et al. Recommendations for the management of hemophagocytic lymphohistiocytosis in adults. Blood 2019;133(23):2465-2477. doi: 10.1182/blood.2018894618 [published Online First: 2019/04/18]

[12.]Sung L, King SM, Carcao M, et al. Adverse outcomes in primary hemophagocytic lymphohistiocytosis. J Pediatr Hematol Oncol 2002;24(7):550-554.

[13.]Gupta AA, Tyrrell P, Valani R, et al. Experience with hemophagocytic lymphohistiocytosis/macrophage activation syndrome at a single institution. J Pediatr Hematol Oncol 2009;31(2):81-84. doi: 10.1097/MPH.0b013e3181923cb4

[14.]Locatelli F, Jordan MB, Allen C, et al. Emapalumab in Children with Primary Hemophagocytic Lymphohistiocytosis. The New England journal of medicine 2020;382(19):1811-1822. doi: 10.1056/NEJMoa1911326 [published Online First: 2020/05/07]

[15.]Ravelli A, Minoia F, Davi S, et al. 2016 Classification Criteria for Macrophage Activation Syndrome Complicating Systemic Juvenile Idiopathic Arthritis: A European League Against Rheumatism/American College of Rheumatology/Paediatric Rheumatology International Trials Organisation Collaborative Initiative. Arthritis \& rheumatology 2016;68(3):566-576. doi: 10.1002/art.39332

[16.]Filipovich $A H$. Hemophagocytic lymphohistiocytosis $(\mathrm{HLH})$ and related disorders. Hematology Am Soc Hematol Educ Program 2009:127-131. doi: 10.1182/asheducation-2009.1.127 [published Online First: 2009/12/17]

[17.]Ahn SS, Yoo BW, Jung SM, et al. Application of the 2016 EULAR/ACR/PRINTO Classification Criteria for Macrophage Activation Syndrome in Patients with Adult-onset Still Disease. The Journal of rheumatology 2017;44(7):996-1003. doi: 10.3899/jrheum.161286

[18.]Jamilloux $Y$, Gerfaud-Valentin $M$, Henry T, et al. Treatment of adult-onset Still's disease: a review. Therapeutics and clinical risk management 2015;11:33-43. doi: 10.2147/TCRM.S64951

[19.]Wang R, Li T, Ye S, et al. Macrophage activation syndrome associated with adult-onset Still's disease: a multicenter retrospective analysis. Clinical rheumatology 2020;39(8):2379-2386. doi: 10.1007/s10067020-04949-0 [published Online First: 2020/03/05]

[20.]Johnson TS, Terrell CE, Millen SH, et al. Etoposide selectively ablates activated T cells to control the immunoregulatory disorder hemophagocytic lymphohistiocytosis. Journal of immunology 2014;192(1):84-91. doi: 10.4049/jimmunol.1302282 [published Online First: 2013/11/22] 


\section{Tables}

Table1. Demographics and clinical characteristics and laboratory findings of refractory AOSD associated MAS patients

\begin{tabular}{|llll|}
\hline & $\begin{array}{l}\text { Etoposide group } \\
\mathrm{n}=17\end{array}$ & $\begin{array}{l}\text { Control group } \\
\mathrm{n}=11\end{array}$ & $\mathrm{P}$ \\
\hline Sex(male/female) & $5 / 12$ & $3 / 8$ & 0.624 \\
\hline Age(Median(p25,p75)) & $33(23-48)$ & $23(20-29)$ & 0.032 \\
\hline Death,n(\%) & $1(5.88 \%)$ & $6(54.55 \%)$ & 0.007 \\
\hline Fever, $\mathrm{n}(\%)$ & $17(100)$ & $11(100)$ & 1.000 \\
\hline Splenomegaly,n(\%) & $12(70.59)$ & $9(81.82)$ & 0.419 \\
\hline Bone marrow & $13(76.47)$ & $8(72.73)$ & 0.581 \\
\hline hemophagocytosis,n(\%) & & & 0.643 \\
\hline White blood cell (×109/L) & $6.43(2.34-16.81)$ & $5.30(1.90-19.30)$ & 0.926 \\
\hline Neutrophil $\left(\times 10^{9} / \mathrm{L}\right)$ & $3.30(1.25-10.88)$ & $4.40(1.34-17.00)$ & 0.677 \\
\hline Hemoglobin $(\mathrm{g} / \mathrm{L})$ & $104(96-119)$ & $113(85-124)$ & 0.547 \\
\hline Platelet $\left(\times 10^{9} / \mathrm{L}\right)$ & $82(49-142)$ & $94(83-103)$ & 0.985 \\
\hline Alanine aminotransferase $(\mathrm{U} / \mathrm{L})$ & $357(164-650)$ & $339(169-873)$ & 0.740 \\
\hline Aspartate aminotransferase $(\mathrm{U} / \mathrm{L})$ & $218(127-492)$ & $253(151-596)$ & 0.675 \\
\hline Triglycerides(mmol/L) & $2.02(1.68-4.06)$ & $3.15(2.03-4.29)$ & 0.109 \\
\hline Fibrinogen(g/L) & $1.40(1.20-2.35)$ & $1.21(0.96-1.91)$ & 0.100 \\
\hline Ferritin(ng/mL)* & $1928(1500-10975)$ & $1500(1500-1500)$ & 0 \\
\hline
\end{tabular}

*Between 2012 to 2017, The upper limit of ferritin test was 1,500 ng/mL in Renji Hospital

Values are presented as the number (\%) or the mean \pm SD

\section{Figures}




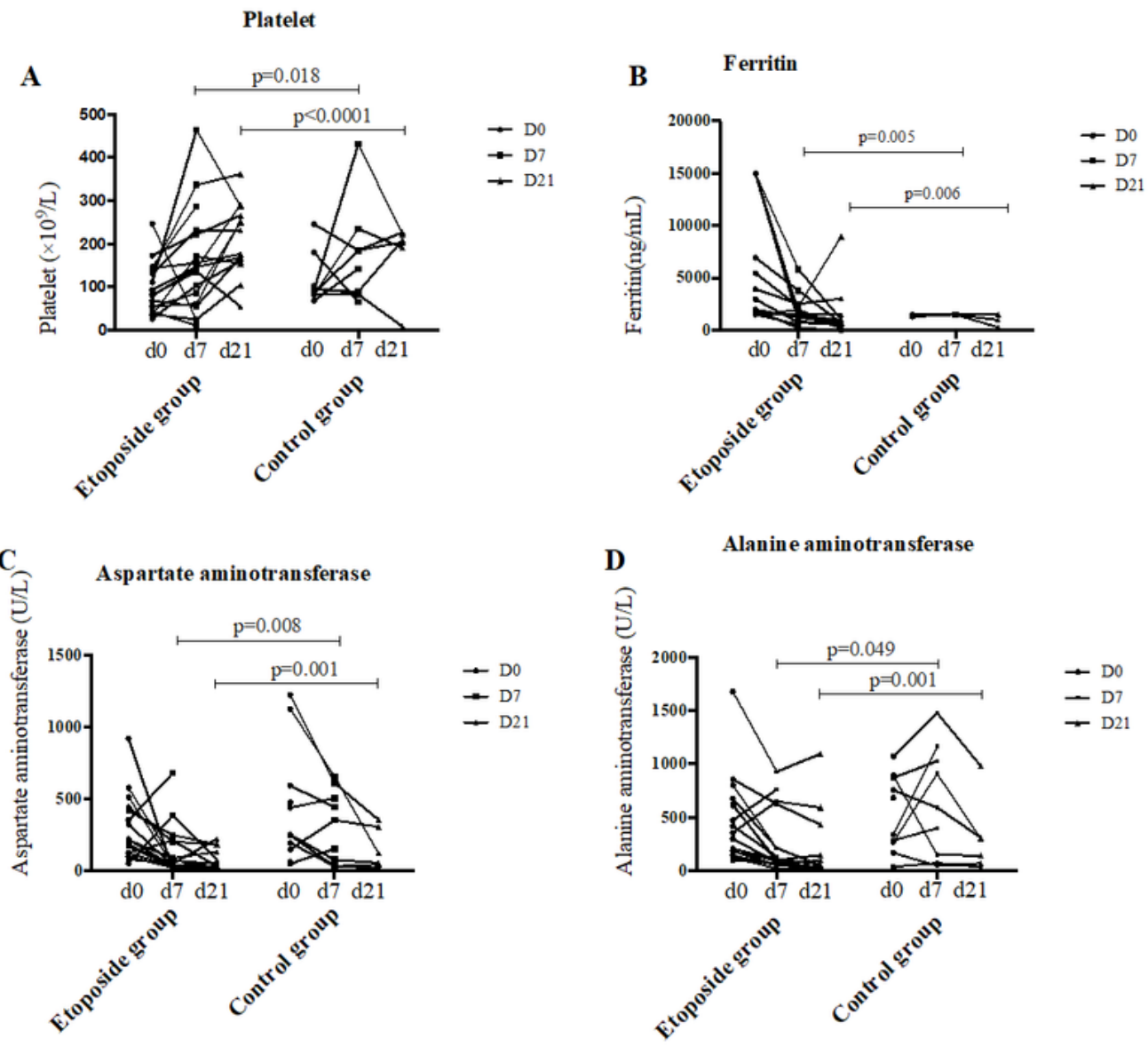

E Survival curve of refractory AOSD associated MAS patients

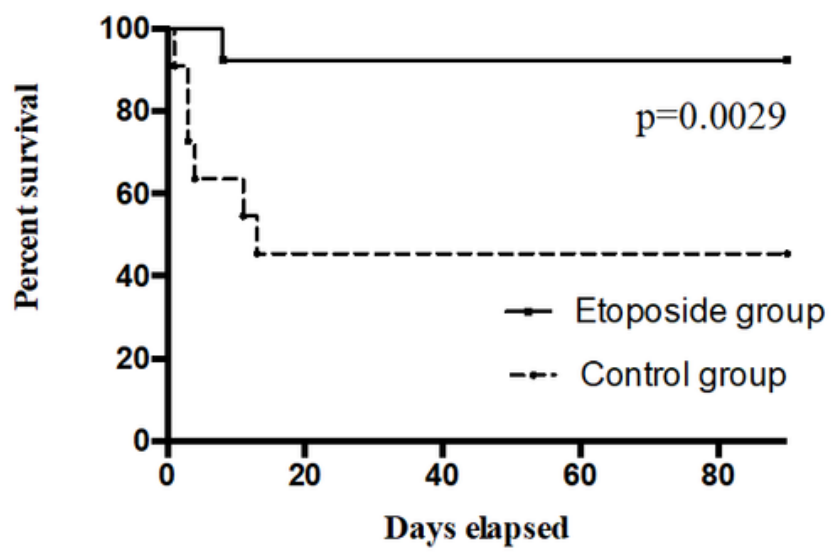

\section{Figure 1}

A-D The use of etoposide remarkably and quickly improved the clinical manifestations and laboratory results. E The 90-day survival rate after the onset of MAS was significantly higher among the patients in the etoposide group than among those in the control group

\section{Supplementary Files}


This is a list of supplementary files associated with this preprint. Click to download.

- supplementarytables.docx 